\title{
Tensor and scalar interactions of neutrinos may lead to observable neutrino magnetic moments
}

\author{
Xun-Jie Xu \\ Max-Planck-Institut für Kernphysik, Postfach 103980, D-69029 Heidelberg, Germany
}

(Received 4 January 2019; published 3 April 2019)

\begin{abstract}
Recently more generalized four-fermion interactions of neutrinos such as tensor and scalar interactions (TSIs) have been extensively studied in response to forthcoming precision measurements of neutrino interactions. We show that due to the chirality-flipping nature, at the one-loop level TSIs typically generate much larger $\left(10^{7}-10^{10}\right)$ neutrino magnetic moments $(\nu \mathrm{MMs})$ than the vector case. For some cases, the large $\nu$ MMs generated by TSIs may reach the known bounds, which implies potentially important interplay between probing TSIs and searching for $\nu \mathrm{MMs}$ in current and future neutrino experiments.
\end{abstract}

DOI: 10.1103/PhysRevD.99.075003

\section{INTRODUCTION}

As neutrino experiments are entering the precision era, searching for new neutrino interactions beyond the Standard Model (BSM) is of increasing importance. In the near future, experiments of coherent neutrino-nucleus scattering ${ }^{1}$ and other types of neutrino scattering ${ }^{2}$ will reach unprecedented sensitivity to various types of BSM neutrino interactions.

Among various BSM interactions considered for neutrinos, the so-called nonstandard interactions (NSIs; see reviews [17-20]), which couple neutrinos $(\nu)$ to other fermions $(\psi)$ by the flavor-changing effective operators $\overline{\nu_{\alpha}} \gamma_{\mu} \nu_{\beta} \bar{\psi} \gamma^{\mu} \psi$, have been extensively studied due to their rich phenomenology in neutrino oscillation. In addition to NSIs which are of the vector form (i.e., containing $\gamma_{\mu}$ between $\bar{\nu}$ and $\nu$ ), recently there has been rising interest in more general interactions [10,21-32] of scalar or tensor forms with the $\gamma_{\mu}$ replaced by $\mathbf{1}$ or $\sigma_{\mu \nu}$, respectively. ${ }^{3}$ From the theoretical point of view, the scalar or tensor interactions

\footnotetext{
${ }^{1}$ First observed by the recent COHERENT experiment [1]. The future experiments include CONUS [2], $\nu$-cleus [3], CONNIE [4], MINER [5], etc.

${ }^{2}$ For example, neutrino scattering at the near detectors [6-12] of long baseline experiments, or at IsoDAR [13,14], LZ [15,16], etc.

${ }^{3}$ More generally, one can have additional $\gamma_{5}$ 's attached, which would form pseudoscalar, axial vector, and $C P$-violating tensor interactions. Hereafter, as a simplified terminology, we will refer to them as scalar, vector, and tensor interactions likewise.

Published by the American Physical Society under the terms of the Creative Commons Attribution 4.0 International license. Further distribution of this work must maintain attribution to the author(s) and the published article's title, journal citation, and DOI. Funded by SCOAP ${ }^{3}$.
}

are as well motivated as the NSIs, since they can all originate from integrating out some BSM bosons. ${ }^{4}$

In this paper, we would like to point out that the scalar or tensor interactions of neutrinos may lead to much larger neutrino magnetic moments $(\nu \mathrm{MMs})$ than the vector interactions. For the vector case, the loop-generated $\nu \mathrm{MM}$ is proportional to the neutrino mass and thus highly suppressed [33-38]. However, for scalar or tensor interactions, due to their chirality-flipping feature, as will be explained later, it is proportional to the mass of $\psi,{ }^{5}$ which is about $10^{7}$ to $10^{10}$ times larger than the neutrino masses. If neutrinos have sizable scalar/tensor interactions at the magnitude that concerns the current neutrino scattering experiments, the large $\nu \mathrm{MMs}$ may reach the known bounds. The connection between scalar/tensor interactions and large $\nu \mathrm{MMs}$ has important implications for future neutrino experiments-if sizable scalar/tensor interactions could be found within the sensitivity of future experiments, then it might imply large, detectable $\nu \mathrm{MMs}$ which would motivate more elaborate experimental searches, and vice versa.

\section{II. $\nu$ MM FROM EFFECTIVE INTERACTIONS}

In what follows, through an explicit but short calculation (depicted in Fig. 1), we will show that $\nu$ MMs generated by scalar/tensor interactions are in general proportional to charged fermion masses instead of neutrino masses. The calculation per se will technically explain the reason. To get

\footnotetext{
${ }^{4}$ Integrating out a vector boson may give rise to NSIs, while integrating out a charged scalar boson may lead to both scalar and tensor interactions-exemplified later in Sec. III.

${ }^{5}$ The idea of obtaining large $\nu$ MMs by avoiding it from being proportional to a neutrino mass is not new and has been discussed widely in the literature; see the review [39] and references therein. For further discussions, see Sec. IV.
} 
a deeper insight into it, after the calculation we will provide an alternative explanation based on fermion chiralities.

We start by considering the following general effective interactions of neutrinos ${ }^{6}(\nu)$ and other fermions $(\psi)$ :

$$
\mathcal{L} \supset G_{X}(\bar{\nu} \Gamma \nu)\left(\bar{\psi} \Gamma^{\prime} \psi\right)
$$

where $\Gamma$ and $\Gamma^{\prime}$ can be any Dirac matrices that keep Eq. (1) Lorentz invariant, including

$$
\mathbf{1}, \quad \gamma_{5}, \quad \gamma_{\mu}, \quad \gamma_{\mu} \gamma_{5}, \quad \sigma_{\mu \nu} \equiv \frac{i}{2}\left[\gamma_{\mu}, \gamma_{\nu}\right]
$$

and their linear combinations (e.g., $\gamma_{\mu}-\gamma_{\mu} \gamma_{5}$ ).

In terms of Feynman diagrams, Eq. (1) is an effective vertex of four fermion lines shown in Fig. 1(a), relevant to elastic neutrino scattering processes that are currently undergoing precision measurement. Given such a diagram, one can close the $\psi$ and $\bar{\psi}$ lines and attach an external photon line to it, which forms a one-loop diagram responsible for $\nu \mathrm{MM}$ generation. The one-loop diagram can be evaluated as follows:

Fig.1(b) $=\int \frac{d^{4} k}{(2 \pi)^{4}} e G_{X} \overline{u_{2}}\left(p_{2}\right) \Gamma u_{1}\left(p_{1}\right) \epsilon^{\mu}(q) \operatorname{tr}_{\text {loop }}$,

where most notations take the standard convention (e.g., $e$ is the coupling constant of $\psi$ to the photon, $\epsilon^{\mu}$ is the photon polarization vector, etc.), all of the momenta have been defined in Fig. 1 with $k \equiv p_{1}-k_{1}=p_{2}-k_{2}$, and $\operatorname{tr}_{\text {loop }}$ stands for the trace of the loop:

$$
\begin{aligned}
\operatorname{tr}_{\text {loop }} & =\operatorname{tr}\left[\frac{1}{\not k_{2}-m_{\psi}} \gamma_{\mu} \frac{1}{\not k_{1}-m_{\psi}} \Gamma^{\prime}\right] \\
& =\frac{\operatorname{tr}\left[\left(\not k_{2}+m_{\psi}\right) \gamma_{\mu}\left(\not k_{1}+m_{\psi}\right) \Gamma^{\prime}\right]}{\left(k_{2}^{2}-m_{\psi}^{2}\right)\left(k_{1}^{2}-m_{\psi}^{2}\right)} .
\end{aligned}
$$

Throughout the calculation, we assume that neutrinos are Dirac particles and leave the case of Majorana neutrinos for later discussion.

The trace in Eq. (5) is crucial to understanding when the generated $\nu \mathrm{MM}$ is proportional to $m_{\psi}$. For simplicity, let us first focus on the scalar interaction $\left(\Gamma=\Gamma^{\prime}=\mathbf{1}\right)$, for which the trace can be easily worked out:

$$
\operatorname{tr}_{\text {loop }}=m_{\psi} \frac{4\left(k_{1}+k_{2}\right)_{\mu}}{\left(k_{2}^{2}-m_{\psi}^{2}\right)\left(k_{1}^{2}-m_{\psi}^{2}\right)} .
$$

\footnotetext{
${ }^{6}$ Here we consider neutrinos in the mass basis and, for simplicity, we focus on one of the three generations. We will discuss the full three-generation framework in the flavor basis in Sec. IV.
}

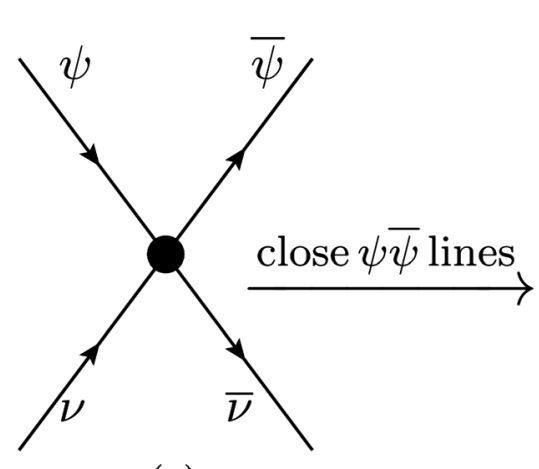

(a)

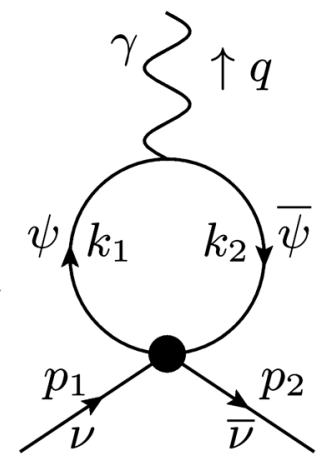

(b)
FIG. 1. Feynman diagrams showing the connection between the effective interactions in Eq. (1) and $\nu$ MMs.

This result can be obtained by noticing that in the numerator of Eq. (5) only the cross terms $\operatorname{tr}\left[m_{\psi} \gamma_{\mu} \not k_{1}+\right.$ $\left.\not k_{2} \gamma_{\mu} m_{\psi}\right]$ are nonzero. This is because the trace of any product containing an odd number of $\gamma$ matrices, such as $\operatorname{tr}\left[k_{2} \gamma_{\mu} \not k_{1}\right]$ and $\operatorname{tr}\left[m_{\psi} \gamma_{\mu} m_{\psi}\right]$, must be zero [40].

Plugging Eq. (6) back into Eq. (3) and integrating out $k$, we should have

Eq. (3) $=m_{\psi} e G_{X} \overline{u_{2}}\left(p_{2}\right)\left[c_{1} p_{1}^{\mu}+c_{2} p_{2}^{\mu}\right] u_{1}\left(p_{1}\right) \epsilon_{\mu}(q)$,

simply by using the Lorentz invariance. Since the quantity between $\overline{u_{2}}$ and $u_{1}$ should be both a Dirac scalar and a Lorentz vector, we must be able to write it as a linear combination of $p_{1}^{\mu}$ and $p_{2}^{\mu}$-here as $c_{1} p_{1}^{\mu}+c_{2} p_{2}^{\mu}$. Furthermore, since Eq. (6) is symmetric under $p_{1} \leftrightarrow p_{2}$, the integral $\int \operatorname{tr}_{\text {loop }} d^{4} k$ should lead to a symmetric result, which implies that $c_{1}=c_{2}$. Indeed, this can be verified by computing the integral manually or using PACKAGE-X [41]. Assuming that the effective vertex has similar UV behavior to the Fermi effective interaction ${ }^{7}$ and $G_{X}^{-1 / 2} \gg m_{\psi} \gg m_{\nu}$, the integral gives

$$
c_{1}=c_{2} \approx \frac{i}{8 \pi^{2}} \equiv c
$$

where " $\approx$ " means that higher-order terms suppressed by $m_{\psi}$ and $m_{\nu}$ are not included.

Using the Gordon identity ${ }^{8}$ and Eq. (8), we can convert Eq. (7) to the magnetic moment form

$$
\text { Fig. 1(b) } \approx c m_{\psi} e G_{X} \overline{u_{2}}\left(p_{2}\right) i \sigma^{\mu \nu} u_{1}\left(p_{1}\right) q_{\nu} \epsilon_{\mu}(q),
$$

\footnotetext{
${ }^{7}$ If $G_{X}$ is a constant at arbitrarily high energies, the integral is divergent. We assume that at low energies $G_{X}$ approximately remains constant while for $k \rightarrow \infty, G_{X}$ decreases as $k^{-2}$. More specifically, we adopt $G_{X} \propto \frac{1}{k^{2}-m^{2}}$, with $m^{2} \sim G_{X}^{-1}$ standing for the energy scale of this transition.

${ }^{8}$ See, e.g., Appendix A of Ref. [42].
} 
which implies the following $\iota \mathrm{MM}$ :

$$
\boldsymbol{\mu}_{\nu} \approx \frac{e G_{X} m_{\psi}}{8 \pi^{2}} \text { (for scalar) }
$$

As one can see, to get $\boldsymbol{\mu}_{\nu} \propto m_{\psi}$, the crucial step in the above calculation is that the trace in Eq. (5) has nonvanishing cross terms (proportional to $m_{\psi}$ ), while all of the other terms are zero. This is true for $\Gamma=\Gamma^{\prime}=\mathbf{1}$. If $\left(\Gamma, \Gamma^{\prime}\right)=\left(\gamma_{\nu}, \gamma^{\nu}\right)$, we would be in the opposite situationthe cross terms become zero, while the other terms are nonzero. A straightforward calculation can confirm that the $\nu \mathrm{MM}$ in this case is approximately proportional to $m_{\nu}$ instead of $m_{\psi}$.

To summarize, whether $\operatorname{tr}\left[\left(m_{\psi} \gamma_{\mu} \not k_{1}+\not k_{2} \gamma_{\mu} m_{\psi}\right) \Gamma^{\prime}\right]$ vanishes or not depends on whether $\Gamma^{\prime}$ consists of an odd or an even number of $\gamma$ matrices. Therefore, for the tensor interaction, we can infer that the result should be proportional to $m_{\psi}$. Indeed, repeating the previous calculation for $\left(\Gamma, \Gamma^{\prime}\right)=\left(\sigma_{\nu \lambda}, \sigma^{\nu \lambda}\right)$ with the same assumptions gives

$$
\boldsymbol{\mu}_{\nu} \approx \frac{e G_{X} m_{\psi}}{2 \pi^{2}}\left[1+\log \left(m_{\psi}^{2} G_{X}\right)\right] \text { (for tensor). }
$$

So far we have technically explained why tensor and scalar interactions could lead to large $\nu$ MMs proportional to $m_{\psi}$. The above argument based on even/odd numbers of $\gamma$ matrices can be more physically interpreted using the concept of chirality flipping.

First, let us examine the chirality of $\nu \mathrm{MM}$,

$$
\begin{aligned}
\mathcal{L}_{\nu \mathrm{MM}} & =\boldsymbol{\mu}_{\nu} \bar{\nu}\left[i \sigma^{\mu \nu} q_{\nu}\right] \nu A_{\mu}, \\
& =\boldsymbol{\mu}_{\nu} \bar{\nu}\left[i \sigma^{\mu \nu} q_{\nu}\right]\left(P_{L}+P_{R}\right) \nu A_{\mu}, \\
& =\boldsymbol{\mu}_{\nu}\left[\overline{\nu_{R}} \sigma^{\mu \nu} \nu_{L}+\overline{\nu_{L}} \sigma^{\mu \nu} \nu_{R}\right] i A_{\mu} q_{\nu},
\end{aligned}
$$

where $P_{L / R} \equiv \frac{1}{2}\left(\mathbf{1} \mp \gamma_{5}\right)$ and $\nu_{L / R} \equiv P_{L / R} \nu$. Equation (14) implies that a $\nu \mathrm{MM}$ itself has to be chirality flipping, i.e., a left-handed neutrino, after participating in the interaction, will turn into a right-handed neutrino, and vice versa.

On the other hand, all vector interactions preserve chirality because

$$
\bar{\nu} \gamma^{\mu} \nu=\overline{\nu_{L}} \gamma^{\mu} \nu_{L}+\overline{\nu_{R}} \gamma^{\mu} \nu_{R}
$$

So to obtain a nonzero $\nu \mathrm{MM}$, we need chirality-flipping sources. One such source is a Dirac neutrino mass term,

$$
m_{\nu} \bar{\nu} \nu=m_{\nu}\left(\overline{\nu_{L}} \nu_{R}+\overline{\nu_{R}} \nu_{L}\right),
$$

which explicitly shows chirality flipping. In addition, as can be checked, tensor or scalar interactions all have the chirality-flipping property.

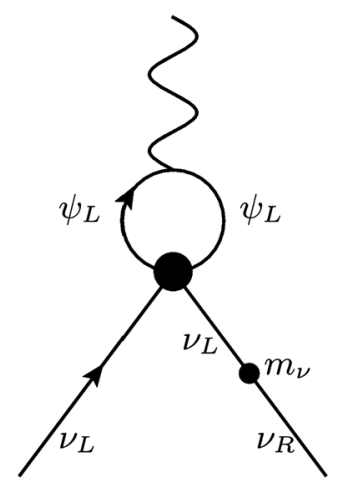

(a): $\boldsymbol{\mu}_{\nu} \propto m_{\nu}$

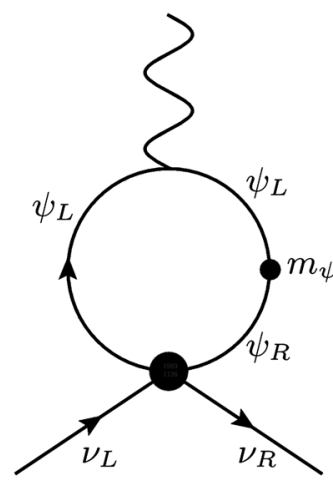

(b): $\boldsymbol{\mu}_{\nu} \propto m_{\psi}$
FIG. 2. Feynman diagrams explaining when $\boldsymbol{\mu}_{\nu}$ are suppressed (a) by the neutrino mass and (b) by the charged lepton mass.

Now let us scrutinize the chirality in the loop diagram. If the four-fermion vertex does not flip chirality (e.g., $\Gamma=\gamma_{\nu}$ and $\Gamma^{\prime}=\gamma^{\nu}$ ), then chirality flipping can only be achieved by $m_{\nu} \bar{\nu} \nu$, as presented in Fig. 2(a). It is interpreted as follows. First, if the left leg is $\nu_{L}$, then the right leg initially has to be $\nu_{L}$ since the four-fermion vertex cannot flip chirality. However, as required by the chirality-flipping property of $\nu \mathrm{MM}$, the right leg eventually should be $\nu_{R}$. So a mass insertion necessarily appears on the right leg to achieve the flipping. In this case, the diagram must be proportional to $m_{\nu}$.

If the four-fermion vertex is of tensor or scalar form [see Fig. 2(b)], then the right leg has the opposite chirality of the left, simply due to the chirality-flipping property of the vertex. So we do not need the mass insertion of $m_{\nu}$. But we should notice that the charged fermion also flips its chirality when passing this vertex, while the photon vertex is not chirality flipping. To accommodate both vertices in one loop, a mass insertion of $m_{\psi}$ is necessary, as marked in Fig. 2(b). In this case, the diagram must be proportional to $m_{\psi}$.

Therefore, we can conclude that if the four-fermion vertex is chirality flipping per se, then it generates $\boldsymbol{\mu}_{\nu} \propto m_{\psi}$; otherwise it leads to $\boldsymbol{\mu}_{\nu} \propto m_{\nu}$. This explains why in our previous calculation $\boldsymbol{\mu}_{\nu} \propto m_{\psi}$ is obtained for tensor and scalar interactions.

\section{A UV COMPLETE EXAMPLE}

The chirality analysis explicates when $\boldsymbol{\mu}_{\nu}$ is proportional to $m_{\psi}$ and when to $m_{\nu}$. The specific values of $\boldsymbol{\mu}_{\nu}$, however, depend on the UV completion of the effective vertex. Below we would like to study a UV complete example which introduces a charged scalar $\phi^{ \pm}$interacting with both left-/right-handed neutrinos $\left(\nu_{L} / \nu_{R}\right)$ and charged leptons $\left(\ell_{L} / \ell_{R}\right)$ :

$$
\mathcal{L} \supset y_{c} \overline{\nu_{L}} \phi^{+} \ell_{R}+y_{s} \overline{\ell_{L}} \phi^{-} \nu_{R}+\text { H.c. }
$$


The above terms could originate from left-right symmetric models (LRSMs) [43-45] $]^{9}$ containing the Yukawa interaction $\left(\overline{\nu_{L}}, \overline{\ell_{L}}\right) \Phi\left(\nu_{R}, \ell_{R}\right)^{T}$, where $\Phi$ is a bidoublet, provided that the charged components in $\Phi$ have generic mass mixing.

Equation (17) can give rise to the four-fermion effective interactions of both scalar and tensor forms if $\phi^{ \pm}$is integrated out:

$$
\mathcal{L}_{\text {eff }}=\frac{y_{c} y_{s}}{m_{\phi}^{2}}\left(\overline{\nu_{L}} \ell_{R}\right)\left(\overline{\ell_{L}} \nu_{R}\right)+\text { H.c. },
$$

which after the Fierz transformation ${ }^{10}$ becomes

$$
\mathcal{L}_{\text {eff }}=-\frac{y_{c} y_{s}}{8 m_{\phi}^{2}}\left(4 \overline{\ell_{L}} \ell_{R} \overline{\nu_{L}} \nu_{R}+\overline{\ell_{L}} \sigma^{\mu \nu} \ell_{R} \overline{\nu_{L}} \sigma_{\mu \nu} \nu_{R}\right)+\text { H.c. }
$$

Given the Yukawa interactions in Eq. (17), we know the specific UV behavior of the effective interactions at high energies. So $\boldsymbol{\mu}_{\nu}$ can be computed without uncertainties caused by UV divergences. There are two diagrams responsible for $\boldsymbol{\mu}_{\nu}$ :

(i) Figure 1(b) with the four-fermion vertex replaced by a $\phi^{ \pm}$mediator.

(ii) A similar diagram as (i) but with the photon coupled to the $\phi^{ \pm}$mediator.

After straightforward loop calculations, the results are

$$
\begin{gathered}
\boldsymbol{\mu}_{\nu}^{(\mathrm{i})}=\frac{e m_{\ell} y_{c} y_{s}}{64 \pi^{2} m_{\phi}^{2}}\left(3+2 \log \frac{m_{\ell}^{2}}{m_{\phi}^{2}}\right), \\
\boldsymbol{\mu}_{\nu}^{(\mathrm{ii})}=-\frac{e m_{\ell} y_{c} y_{s}}{64 \pi^{2} m_{\phi}^{2}},
\end{gathered}
$$

corresponding to the contributions of (i) and (ii), respectively. So in this model the total contribution to the $\nu \mathrm{MM}$ is

$$
\boldsymbol{\mu}_{\nu}=\boldsymbol{\mu}_{\nu}^{(\mathrm{i})}+\boldsymbol{\mu}_{\nu}^{(\mathrm{ii})}=\frac{e m_{\ell} y_{c} y_{s}}{32 \pi^{2} m_{\phi}^{2}}\left(1+\log \frac{m_{\ell}^{2}}{m_{\phi}^{2}}\right) .
$$

This is consistent with our previous discussions based on the effective operators [cf. Eqs. (10) and (11)]. Taking $G_{X} \sim y_{c} y_{s} /\left(8 m_{\phi}^{2}\right)$, we can see that the effective and the UV complete results agree at the same order of magnitude, while the difference is understandable due to different UV details.

\footnotetext{
${ }^{9}$ Since $\nu_{R}$ appears as an external fermion line in Fig. 2, the canonical LRSM in which right-handed neutrinos are heavy states cannot be applied here.

${ }^{10}$ To use the chiral form, see Eqs. (2.6) and (2.7) of Ref. [46].
}

\section{DISCUSSION AND CONCLUSION}

Throughout the paper, we have considered only the case of Dirac neutrinos. For Majorana neutrinos, our conclusions would be similar but would need slight modification. As is well known, Majorana neutrinos can have only transition magnetic moments, meaning that the corresponding term $\bar{\nu}_{i} \sigma^{\mu \nu} \nu_{j} q_{\nu} A_{\mu}$ may exist only if $i \neq j(i, j=1,2,3$ denote the mass eigenstates of neutrinos; $\nu \equiv \nu_{L}+\nu_{L}^{c}$ is a Majorana spinor such that $\nu=\nu^{c}$ ). Viewed from fermionic degrees of freedom, the transition from $\nu_{i} \rightarrow \nu_{j}$ is essentially equivalent to the aforementioned chirality flipping, as the initial and final neutrinos are two different Weyl spinors. Therefore, for Majorana neutrinos, we simply need the replacement $\left(\nu_{R}, \nu_{L}\right) \rightarrow\left(\nu_{L i}^{c}, \nu_{L j}\right)$ in the above analyses.

The analyses in this paper can be readily extended to include three flavors. First, Eq. (1) can be modified to the flavor-dependent form

$$
\mathcal{L} \supset G_{X}^{\alpha \beta}\left(\overline{\nu_{\alpha}} \Gamma \nu_{\beta}\right)\left(\bar{\psi} \Gamma^{\prime} \psi\right),
$$

where $\alpha, \beta=e, \mu, \tau$ are flavor indices. Then since we know that, for tensor and scalar interactions, neutrino masses make negligible contributions to $\nu \mathrm{MMs}$, neutrinos can be treated as massless particles in the calculation, which would lead to flavor-dependent $\boldsymbol{\mu}_{\nu}^{\alpha \beta}$ in Eqs. (10) and (11) with only $G_{X}$ replaced by $G_{X}^{\alpha \beta}$. Note that many experimental measurements actually produce constraints on combinations of some $\boldsymbol{\mu}_{\nu}^{\alpha \beta}$. For example, $\nu_{e}-e$ scattering experiments with negligible baselines are sensitive to the effective magnetic moment of $\nu_{e}$ below [47]:

$$
\boldsymbol{\mu}_{\nu_{e}}^{2}=\sum_{\beta}\left|\boldsymbol{\mu}_{\nu}^{e \beta}\right|^{2}
$$

For solar neutrino experiments, the effective magnetic moment being constrained is [39]

$$
\boldsymbol{\mu}_{S}^{2}=\sum_{j, k=1}^{3}\left|U_{e k}^{M}\right|^{2}\left|\boldsymbol{\mu}_{\nu}^{j k}\right|^{2},
$$

where $U_{e k}^{M}$ is the effective neutrino mixing with the matter effect included, and $\boldsymbol{\mu}_{\nu}^{j k}$ is the mass-basis form of $\boldsymbol{\mu}_{\nu}^{\alpha \beta}$. In addition, for plasmon decay $\left(\gamma^{*} \rightarrow \bar{\nu} \nu\right)$ [48], one can define the following effective magnetic moment,

$$
\boldsymbol{\mu}_{\gamma}^{2}=\sum_{j, k=1}^{3}\left|\boldsymbol{\mu}_{\nu}^{j k}\right|^{2}=\sum_{\alpha, \beta}\left|\boldsymbol{\mu}_{\nu}^{\alpha \beta}\right|^{2},
$$

which is useful in interpreting the astrophysical bounds.

The values of $\boldsymbol{\mu}_{\nu}$ given by Eqs. (10) and (11) depend on the UV completion of the effective vertices. Being model dependent implies that $\boldsymbol{\mu}_{\nu}$ could be much smaller or larger 
than Eqs. (10) and (11) in particular models. For example, if it is UV completed by a neutral scalar $\phi$ with Yukawa interactions $\bar{\nu} \nu \phi$ and $\bar{\psi} \psi \phi$, then the loop diagram naively gives zero $\boldsymbol{\mu}_{\nu}$. However, since it breaks $S U(2)_{L}$, usually this model is a fragment of some more complete gauge invariant models, in which $\phi$ would be the neutral component of a Higgs multiplet and would be accompanied with charged scalar bosons. The full calculation, including contributions from charged bosons, may again lead to large nonzero $\boldsymbol{\mu}_{\nu}$.

Taking Eqs. (10) and (11) as the typical values of $\boldsymbol{\mu}_{\nu}$ generated by the effective tensor and scalar interactions, we plot them in Fig. 3 together with terrestrial (TEXONO [49], Borexino [50], GEMMA [51], and LZ equipped with an intensive ${ }^{51} \mathrm{Cr}$ radiative source [15]) and astrophysical [48] bounds. Currently the effective coupling $G_{X}$ can be constrained by various elastic neutrino scattering data from CHARM II, LSND, TEXONO, Borexino, COHERENT, etc. In general, these experiments have $G_{X}$ sensitivity ranging from $0.1 G_{F}$ to $1 G_{F}[22,25,30,52]$, depending on the neutrino flavors, the charged fermion $\psi$, the specific forms of new interactions, etc. With these details involved and the uncertainties of theoretical predictions due to the UV incompleteness, here we refrain from more specific discussions and show merely two bands (red) of $G_{X}=$ $0.1 G_{F}-1 G_{F}$ in Fig. 3. In the future, the DUNE near detector and some reactor-based coherent neutrino scattering experiments may significantly improve the sensitivity by 1 or 2 orders of magnitude [10,23].

The significance of Fig. 3 showing the red bands and the blue limits in the same windows is manifold. If, e.g., $G_{X}=0.1 G_{F}$ for tensor interactions had been probed in neutrino-electron scattering experiments, it would imply a large $\nu \mathrm{MM}\left(\boldsymbol{\mu}_{\nu} \sim 10^{-12} \mu_{B}\right)$ that could be observed by improving $\nu \mathrm{MM}$ experiments by 1 order of magnitude.

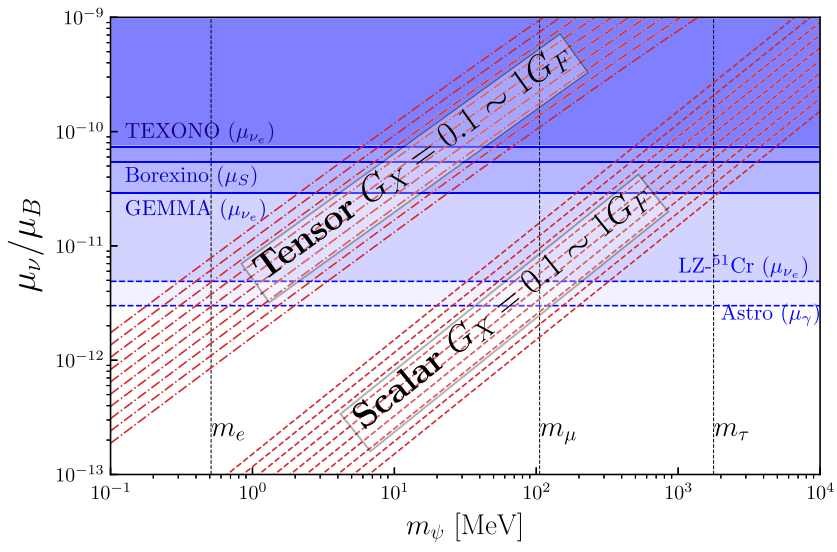

FIG. 3. $\boldsymbol{\mu}_{\nu}$ predicted by tensor and scalar interactions (red lines) compared with experimental bounds (blue). Here the GEMMA, TEXONO, and $\mathrm{LZ}_{-}{ }^{51} \mathrm{Cr}$ bounds are only for the effective magnetic moment $\boldsymbol{\mu}_{\nu_{e}}$ defined in Eq. (24); the Borexino bound is based on solar neutrinos which should be applied to $\boldsymbol{\mu}_{S}$ given in Eq. (25); and the astrophysical (Astro) bound should be applied to $\boldsymbol{\mu}_{\gamma}$ in Eq. (26).
In addition, since the same coupling strength for $\psi=\mu$ and $\tau$ would lead to too large $\boldsymbol{\mu}_{\nu}$, it would imply that in model building, $G_{X}$ for these two flavors must be suppressed, which is of theoretical importance. On the other hand, if in the future we reach much more solid and stringent bounds on $\boldsymbol{\mu}_{\nu}$ (currently LZ- ${ }^{51} \mathrm{Cr}$ is only a proposal and the astrophysical bound could be altered in nonstandard scenarios), it will disprove the presence of sizable tensor and scalar interactions, which is still of importance for both experimental searches and theoretical model building.

The last comment concerns neutrino masses. It has been commonly discussed in the literature (reviewed in Ref. [39]) that the new physics leading to large $\nu \mathrm{MMs}$ usually generates too large neutrino masses. This can be understood by simply noticing that in the absence of chirality-flipping interactions the generated $\nu \mathrm{MM}$ is proportional to $m_{\nu}$. There have been various approaches, however, to get a large $\nu \mathrm{MM}$ while keeping $m_{\nu}$ small. One possibility is to avoid it from being proportional to $m_{\nu}$, which has been discussed in Refs. [34,38,53-61]. For example, in the left-right symmetric model with Dirac neutrinos, $\boldsymbol{\mu}_{\nu} \propto m_{\ell}$ can be obtained [see, e.g., Eq. (2.29) in [38] ] via the charged current (CC) interaction $\bar{\ell}_{L} \gamma^{\mu} \nu_{L} W_{L \mu}^{-}$ and its right-handed partner $\bar{\ell}_{R} \gamma^{\mu} \nu_{R} W_{R \mu}^{-}$, where $W_{L}^{ \pm}$and $W_{R}^{ \pm}$are the charged gauge bosons of $S U(2)_{L}$ and $S U(2)_{R}$ with small mass mixing. From the point of view of effective interactions adopted in this paper, it is straightforward to understand the result. The left- and right-handed CC interactions with mixing can give rise to the effective interaction $\left(\bar{\ell}_{L} \gamma^{\mu} \nu_{L}\right)\left(\bar{\nu}_{R} \gamma^{\mu} \ell_{R}\right)$, which after the Fierz transformation becomes a chirality-flipping scalar interaction $2\left(\bar{\nu}_{R} \nu_{L}\right)\left(\bar{\ell}_{L} \ell_{R}\right)$. This should lead to $\boldsymbol{\mu}_{\nu} \propto m_{\ell}$ according to our conclusion on such scalar interactions. Therefore, the calculation in the previous studies confirms our conclusion on the effective interactions. In addition to this model, there are various other models proposed for large $\nu \mathrm{MMs}$ [62-71]. Although building models for large $\nu \mathrm{MMs}$ is not the focus of this paper, our conclusion indicates that one may preferably introduce chirality-flipping interactions to obtain large $\nu \mathrm{MMs}$ because in this situation, $\boldsymbol{\mu}_{\nu}$ is proportional to $m_{\psi}$ instead of $m_{\nu}$, and the generation of $\nu$ MMs can be detached from the generation of neutrino masses.

In conclusion, our analysis reveals that large $\nu$ MMs may be potentially related to sizable tensor and scalar interactions, and vice versa. The experimental and theoretical significance of the interplay will be explored in further studies.

\section{ACKNOWLEDGMENTS}

X.-J.X would like to thank Evgeny Akhmedov and Alexei Smirnov for many helpful conversations on $\nu \mathrm{MMs}$, Robert Shrock for discussions on $\nu \mathrm{MMs}$ in the left-right symmetric model, and especially Rabindra Mohapatra for insightful discussions on our previous work [25] which gradually developed into the initial idea of this work. 
[1] D. Akimov et al. (COHERENT Collaboration), Science 357, 1123 (2017).

[2] W. Maneschg, DOI: 10.5281/zenodo.1286927.

[3] R. Strauss et al., Eur. Phys. J. C 77, 506 (2017).

[4] A. Aguilar-Arevalo et al. (CONNIE Collaboration), J. Phys. Conf. Ser. 761, 012057 (2016).

[5] G. Agnolet et al. (MINER Collaboration), Nucl. Instrum. Methods Phys. Res., Sect. A 853, 53 (2017).

[6] B. Wang, J. Bian, T. E. Coan, S. Kotelnikov, H. Duyang, and A. Hatzikoutelis (NO $\nu$ A Collaboration), J. Phys. Conf. Ser. 888, 012123 (2017).

[7] J. Bian, arXiv:1710.03428.

[8] A. Falkowski, G. Grilli di Cortona, and Z. Tabrizi, J. High Energy Phys. 04 (2018) 101.

[9] P. deNiverville and C. Frugiuele, arXiv:1807.06501.

[10] I. Bischer and W. Rodejohann, Phys. Rev. D 99, 036006 (2019).

[11] P. Bakhti, Y. Farzan, and M. Rajaee, arXiv:1810.04441.

[12] P. Ballett, M. Hostert, S. Pascoli, Y. F. Perez-Gonzalez, Z. Tabrizi, and R. Zukanovich Funchal, J. High Energy Phys. 01 (2019) 119.

[13] J. M. Conrad, M. H. Shaevitz, I. Shimizu, J. Spitz, M. Toups, and L. Winslow, Phys. Rev. D 89, 072010 (2014).

[14] M. Abs et al., arXiv:1511.05130.

[15] P. Coloma, P. Huber, and J. M. Link, J. High Energy Phys. 11 (2014) 042.

[16] D. S. Akerib et al. (LZ Collaboration), arXiv:1509.02910.

[17] S. Davidson, C. Pena-Garay, N. Rius, and A. Santamaria, J. High Energy Phys. 03 (2003) 011.

[18] T. Ohlsson, Rep. Prog. Phys. 76, 044201 (2013).

[19] Y. Farzan and M. Tortola, Front. Phys. 6, 10 (2018).

[20] I. Esteban, M. C. Gonzalez-Garcia, M. Maltoni, I. MartinezSoler, and J. Salvado, J. High Energy Phys. 08 (2018) 180.

[21] K. J. Healey, A. A. Petrov, and D. Zhuridov, Phys. Rev. D 87, 117301 (2013); 89, 059904(E) (2014).

[22] B. Sevda, M. Deniz, S. Kerman, L. Singh, H. T. Wong, and M. Zeyrek, Phys. Rev. D 95, 033008 (2017).

[23] M. Lindner, W. Rodejohann, and X.-J. Xu, J. High Energy Phys. 03 (2017) 097.

[24] L. Heurtier and Y. Zhang, J. Cosmol. Astropart. Phys. 02 (2017) 042.

[25] W. Rodejohann, X.-J. Xu, and C. E. Yaguna, J. High Energy Phys. 05 (2017) 024.

[26] D. K. Papoulias and T. S. Kosmas, Phys. Rev. D 97, 033003 (2018).

[27] G. Magill and R. Plestid, Phys. Rev. D 97, 055003 (2018).

[28] Y. Farzan, M. Lindner, W. Rodejohann, and X.-J. Xu, J. High Energy Phys. 05 (2018) 066.

[29] Y. Yang and J. P. Kneller, Phys. Rev. D 97, 103018 (2018).

[30] D. Aristizabal Sierra, V. De Romeri, and N. Rojas, Phys. Rev. D 98, 075018 (2018).

[31] V. Brdar, W. Rodejohann, and X.-J. Xu, J. High Energy Phys. 12 (2018) 024.

[32] A. Błaut and W. Sobków, arXiv:1812.09828.

[33] S. T. Petcov, Yad. Fiz. 25, 1336 (1977) [Sov. J. Nucl. Phys. 25, 340 (1977)].

[34] W. J. Marciano and A. I. Sanda, Phys. Lett. 67B, 303 (1977).

[35] B. W. Lee and R. E. Shrock, Phys. Rev. D 16, 1444 (1977).

[36] K. Fujikawa and R. Shrock, Phys. Rev. Lett. 45, 963 (1980).
[37] P. B. Pal and L. Wolfenstein, Phys. Rev. D 25, 766 (1982).

[38] R. E. Shrock, Nucl. Phys. B206, 359 (1982).

[39] C. Giunti and A. Studenikin, Rev. Mod. Phys. 87, 531 (2015).

[40] M. E. Peskin and D. V. Schroeder, An Introduction to Quantum Field Theory (Addison-Wesley, Reading, MA, 1995), p. 805.

[41] H. H. Patel, Comput. Phys. Commun. 197, 276 (2015).

[42] C. Giunti and C. W. Kim, Fundamentals of Neutrino Physics and Astrophysics (Oxford University Press, New York, 2007), pp. 64-66.

[43] J. C. Pati and A. Salam, Phys. Rev. D 10, 275 (1974); 11, 703(E) (1975).

[44] R. N. Mohapatra and J. C. Pati, Phys. Rev. D 11, 2558 (1975).

[45] G. Senjanovic and R. N. Mohapatra, Phys. Rev. D 12, 1502 (1975).

[46] I. Bischer, W. Rodejohann, and X.-J. Xu, J. High Energy Phys. 10 (2018) 096.

[47] K. A. Kouzakov and A. I. Studenikin, Phys. Rev. D 95, 055013 (2017); 96, 099904(E) (2017).

[48] G. G. Raffelt, Phys. Rep. 320, 319 (1999).

[49] H. T. Wong et al. (TEXONO Collaboration), Phys. Rev. D 75, 012001 (2007).

[50] C. Arpesella et al. (Borexino Collaboration), Phys. Rev. Lett. 101, 091302 (2008).

[51] A. G. Beda, V. B. Brudanin, V. G. Egorov, D. V. Medvedev, V. S. Pogosov, M. V. Shirchenko, and A. S. Starostin, Adv. High Energy Phys. 2012, 1 (2012).

[52] M. Lindner, F. S. Queiroz, W. Rodejohann, and X.-J. Xu, J. High Energy Phys. 05 (2018) 098.

[53] R. Shrock, Phys. Rev. D 9, 743 (1974).

[54] J. E. Kim, Phys. Rev. D 14, 3000 (1976).

[55] M. A. B. Beg, W. J. Marciano, and M. Ruderman, Phys. Rev. D 17, 1395 (1978).

[56] M. J. Duncan, J. A. Grifols, A. Mendez, and S. U. Sankar, Phys. Lett. B 191, 304 (1987).

[57] J. Liu, Phys. Rev. D 35, 3447 (1987).

[58] S. Rajpoot, Phys. Lett. B 237, 77 (1990).

[59] M. Czakon, J. Gluza, and M. Zralek, Phys. Rev. D 59, 013010 (1998).

[60] M. Nemevsek, G. Senjanovic, and V. Tello, Phys. Rev. Lett. 110, 151802 (2013).

[61] O. M. Boyarkin and G. G. Boyarkina, Phys. Rev. D 90, 025001 (2014).

[62] M. B. Voloshin, Yad. Fiz. 48, 804 (1988) [Sov. J. Nucl. Phys. 48, 512 (1988)].

[63] B. Alles and F. Giuliani, Nucl. Phys. B339, 1 (1990).

[64] S. M. Barr, E. M. Freire, and A. Zee, Phys. Rev. Lett. 65, 2626 (1990).

[65] S. M. Barr and E. M. Freire, Phys. Rev. D 43, 2989 (1991).

[66] K. S. Babu and R. N. Mohapatra, Phys. Rev. D 43, 2278 (1991).

[67] N. G. Deshpande and P. B. Pal, Phys. Rev. D 45, 3183 (1992).

[68] K. S. Babu, D. Chang, W.-Y. Keung, and I. Phillips, Phys. Rev. D 46, 2268 (1992).

[69] D. Chang, W.-Y. Keung, and I. Phillips, Int. J. Mod. Phys. A 09, 2013 (1994).

[70] M. Frank, Phys. Lett. B 477, 208 (2000).

[71] M. Lindner, B. Radovcic, and J. Welter, J. High Energy Phys. 07 (2017) 139. 